\title{
Keratocyst Multimodal Management in Maxillofacial Surgery: Case Report
}

\author{
Fernández Álvarez Ángel Yair ${ }^{1}$, Elizalde Monroy Martín² and Juárez \\ Rebollar Alejandra Giselle ${ }^{1 *}$ \\ ${ }^{1}$ Resident of Maxillofacial Surgery, National Medical Center, Mexico \\ ${ }^{2}$ Maxillofacial Surgeon, National Medical Center, Mexico
}

*Corresponding author: Juárez-Rebollar Alejandra Giselle, Resident of Maxillofacial Surgery, National Medical Center, Mexico, Tel: 5556276900; Email: giselle_juarez@hotmail.com

\section{Case Report \\ Volume 5 Issue 3}

Received Date: August 03, 2020

Published Date: August 12, 2020

DOI: $10.23880 /$ oajds-16000267

\section{Abstract}

Keratocysts are considered benign lesions, however, as has been reported in many works by different authors, it has been considered aggressive due to its expansion and high recurrence rate. Currently, numerous articles have been published worldwide on different studies for this type of pathology, which propose different treatment alternatives, ranging from conservative management, for example the use of adjuvants, chelators, cryotherapy, decompression, use of decompressive valves, etc. And surgical treatments among these are those carried out with local anesthesia and / or considered minor surgeries such as enucleations, surgical washes and curettage, of which block or extensive resection with or without reconstruction that can be done at the same time are mentioned Surgical or subsequent, so it was decided to carry out a combination of different treatments with which there is literature reporting their efficacy in order to provide a better prognosis to the patient and to avoid, if possible, achieving wide resections. Therefore, a representative case of a patient diagnosed with Keratocyst, treated in Maxillofacial Service of Specialty Hospital, National Medical Center, XXI Century, IMSS. With multimodal treatment, using different decompressive and reconstruction means is presented, resulting in the eradication of two large-sized lesions and without recurrence two years after their management.

Keywords: Keratocyst; Odontogenic Cysts; Enucleation; Mandible

\section{Introduction}

Among the historical background of the keratocyst, the controversy and name change as well as its classification stand out. For example in 1876 the odontogenic keratocyst (QO) was found, later in 1956 Phillipsen classified it and in 1963 Pindborg and Hansen describe it as a welldefined solitary lesion surrounded by a thin cortex $[1,2]$. Subsequently in 2005, it was reclassified by the World Health Organization (WHO) as a keratocystic odontogenic tumor, emphasizing the high recurrence rate, in addition to highlighting its aggressive clinical behavior and which may or may not be associated with any syndrome (for example Gorlin Goltz) this, due to the mutations that occur in the tumor suppressor gene PTCH. Finally, in the year of 2017, the WHO in its 4th edition again names it Odontogenic Keratocyst
(Q0) [3], since it was shown during many studies and years that the mutation of the PTCH gene could also be found in non-neoplastic lesions, including mentioning its appearance in dentigerous cysts [4]. Noting that in various reports and publications different authors from different places show you that with management such as marsupialization they help to resolve it and if it really were a neoplastic process, it would not resolve with such a procedure because it is not compliant [5-7]. Clinically it can be seen unilocular or multilocular radiolucency, most frequently in the posterior mandible, expansion of the buccal and lingual plates occurs late with this injury, can cause lingual plate perforation, recurrence rate of from $25 \%$ to $60 \%$ after local enucleation [8]. Some differential diagnoses of Keratocysts include, dentigerous cysts, ameloblastomas, root cysts, simple bone cysts, and central giant cell granulomas [9]. Histologically, 
it is characterized by epithelial cell proliferation, infiltrative growth, uniformly thin epithelial lining of six to eight layers Cellular, fibrous connective tissue epithelial lifting and formation of crevices, [10] corrugated parakeratinized epithelium $[9,10]$. A palisade basal layer with columnar to cuboidal cells [11] with keratin material in the cystic cavity $[9,10]$. In matter of management and treatment For decades it has been controversial, however it has been unified that depending on the size and characteristics of the lesion, the one that the surgeon considers to be the most appropriate for the patient will be adapted. To mention some treatments and management are marsupialization, decompression, use of valves [12], enucleation, peripheral ostectomy, physicochemical treatment (chemical treatment with Carnoy's solution and Physical treatment with cryotherapy) and more aggressive such as resection with $1-\mathrm{cm}$ margins $[8,13]$.

\section{Case Report}

The case of a 46-years-old female patient is presented, who goes to the Maxillofacial Surgery service of the Specialty Hospital of the National Medical Center, XXI Century, IMSS, for evaluation; She is referred by a dentist from the medical staff, who performs a radiograph, in the molar region, two images multilocular, finding a lesion referred to in the delivery note as: possible cyst vs. tumor to be determined. Among the patient's personal pathological history, reports: penicillin allergy, denies smoking, and chronic-degenerative, traumatic, transfusion, and surgery were also questioned and denied. The symptoms were occasional of the "dental pain" type that was variable in relation to the temperature of the liquids and / or food consistency that the patient ingested. Clinically, there was no increase in volume on the face (Figure 1) or changes in the mucosa, no mobility, slight pain on percussion in the right lower third molar, some old dental restorations without alteration, the rest without data indicating pathology. Therefore, computed tomography is performed for clinical-imaging correlation. The coronal and sagittal sections show the presence of two lesions, one at the level of the mandibular branch and the other on the mandibular body, both on the right side, well defined, with a hyper-dense halo compared to the rest of the mandibular medullary bone. Observing the preservation of the mandibular basal and a relation of approximately $3 \mathrm{~mm}$ with respect to the sigmoid notch (Figures $2 a-2 c$ ).

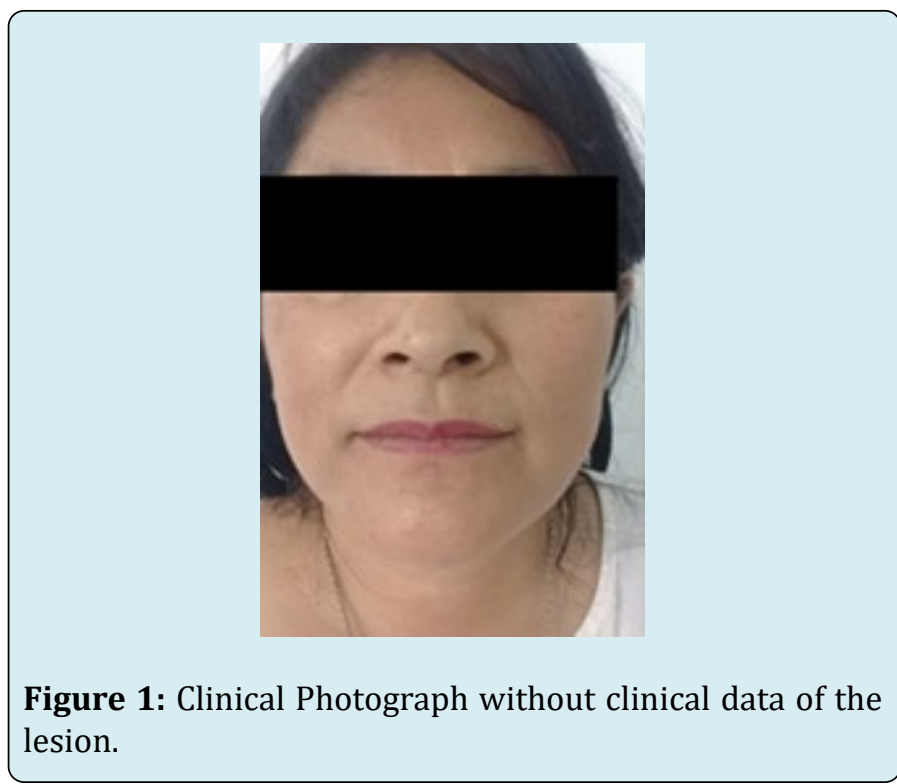

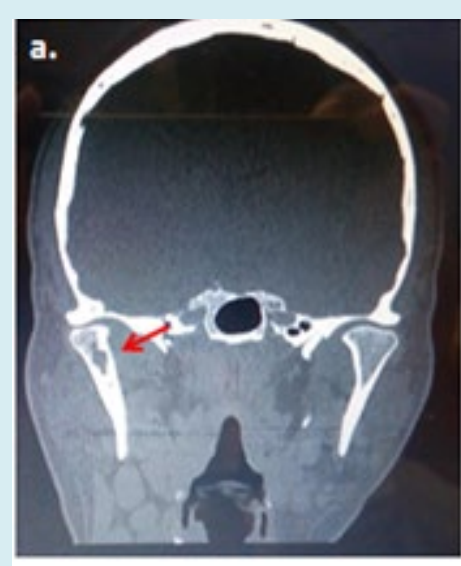
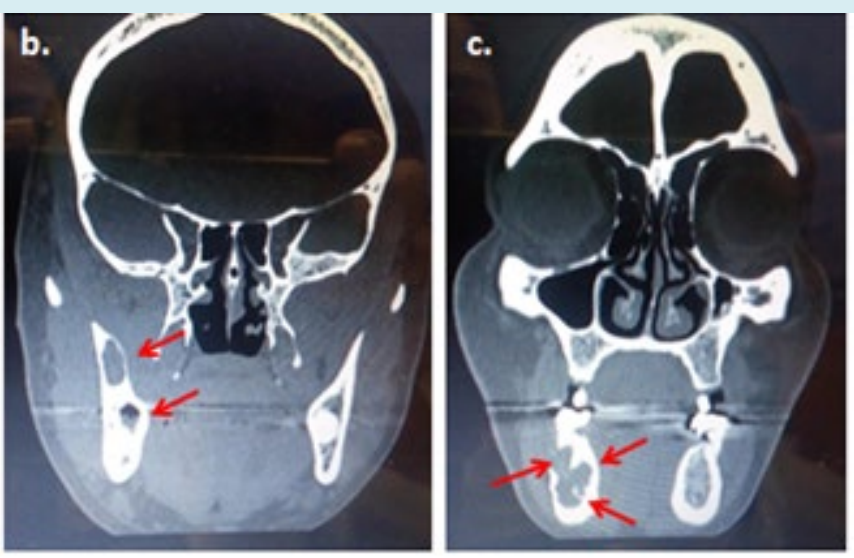

Figures 2a-2c: Coronal section with lesion at the condyle level, below the sigmoid notch (red arrow), b. Coronal section with the presence of two lesions, one on the branch and the other on the mandibular body, both on the right side (red arrow), c. saggital slice with multiocular image on the right mandibular body (red arrow). 
So it was decided to perform an incisional biopsy in the molar region under local anesthesia and sent to pathology for study. Reporting fragmented odontogenic keratocyst with acute and chronic inflammation. Therefore, when corroborating with the image, it was decided to first perform a curettage with cryotherapy (based on ethyl chloride) and placing a decompression valve made of silicone (Figure 3 ) in order to reduce the size of the lesions while remaining as a closed chimney-type wound and daily mouthwashes with $0.12 \%$ chlorhexidine are carried out by the patient with periodic review every week thereafter every 15 days until every month for three months, it is decided to perform a curettage and place lyophilized bone supported by a titanium mesh in the right mandibular body in order to perform the surgical site and contain the bone graft;



Figure 3: Silicone decompression valves with yellow arrows; a. Mandibular branch \& b. Mandibular body.

it is observed in the edges the formation of bone tissue with persistence of some areas suggestive of cystic lesion, radiographic control (Figure 4) is performed finding improvement in the lesion of the mandibular branch observing bone neoformation, however it is decided to remove the titanium mesh since in the mandibular body An apparent persistence of the lesion is observed in the panoramic radiograph, so a new curettage (Figure 5) is performed and a decompressive valve is placed again in the mandibular body aiming at the reduction of the Keratocyst, the mouthwashes with chlorhexidine are again indicated and maintained with controls periodicals of review, finally, a control radiograph was performed without showing recurrence of the lesion. Clinically, one and a half years later, a scar tissue was observed in the incision area (Figure 6) of the surgical procedure, asymptomatic patient, with no clinical or radiographic data of recurrence of the injury.

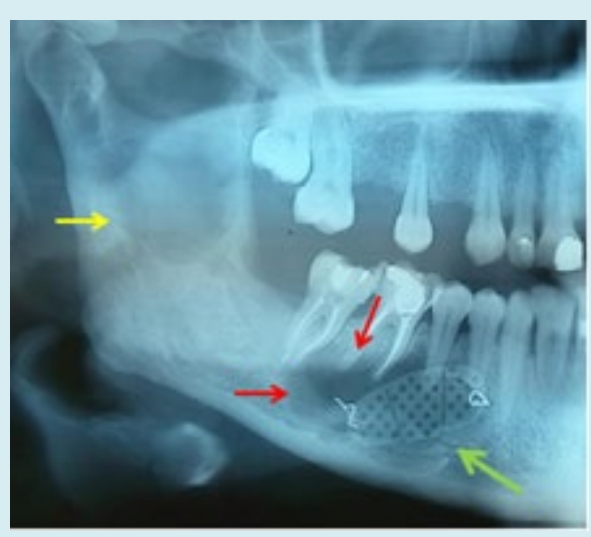

Figure 4: The formation of bone in the mandibular branch (yellow arrow), persistence of the lesion (red arrows) and titanium mesh (green arrow) are observed.

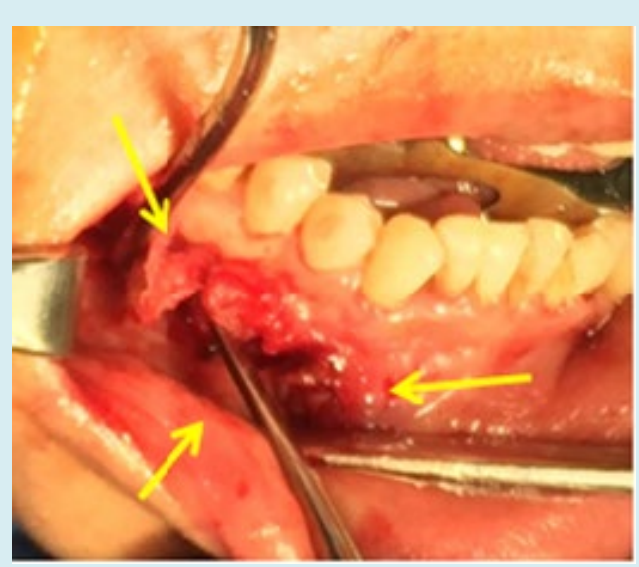

Figure 5: Curtteage of mandibular body injury and removal of titanium mesh (yellow arrows).

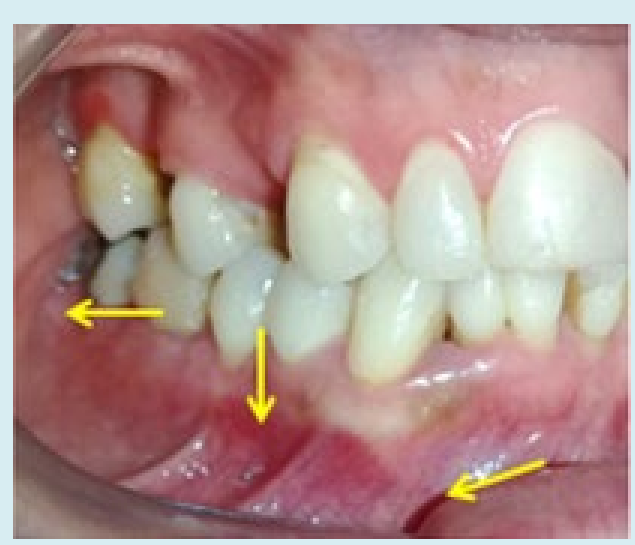

Figure 6: Control clinical photograph at one and a half years, postoperative scar tissue is observed with no pathological injury data. 


\section{Discussion}

Keratocysts as mentioned in different studies have clinical relevance due to the variety of management and treatments that have been used by different surgeons, highlighting in all the studies the importance that must be had in the clinical and radiographic control of patients, as well as the Always consider as part of the pre-surgical protocol to perform a biopsy and send the sample to Pathology so that the histopathological study can be carried out and verify that the diagnosis is Keratocyst, as mentioned in the Clinical Guidelines for Oral and Maxillofacial Surgery in North America [8], or as mentioned by authors such as Pogrel [6] or Juarez [12], and different treatment as mentioned by Wakolbinger [5], Motwani [9] or Nair, et al. [13], among many others. The different differential entities of Keratocysts must be taken into account, as mentioned by Motwani, et al. [9], with histopathological information, better management and treatment can be provided, since their characteristics of each lesion and histopathology represent the basis for deciding between management and therapeutic, thereby avoiding complications, recurrence and / or poor management of patients. We agree with the variety of therapeutics tried and tested by different surgeons, considering the results reported by different authors. Well, having a broad overview of management and therapy helps to make a more appropriate and individualized decision for each patient.

\section{Conclusion}

Keratocysts are odontogenic cysts, one of the most frequent, with clinical similarities to other odontogenic lesions, however histologically and radiographically different, since these studies determine the definitive diagnosis and thus be able to decide between the most appropriate management for each patient. The case presented is an example that notall multiple Keratocysts should be associated with syndromes, the versatility that was had in being able to decide and use different treatments simultaneously and consecutively with excellent results was also demonstrated, thus showing that not all lesions, not all Keratocysts, nor all patients, will respond in the same way. The period of treatment, control and evolution was long-term, since in total it was approximately a year and a half that had the patient with variable and active management and therapy; highlighting that although currently there are no lesions or recurrence, it remains in control, since different articles and literature report the high percentage of recurrence at 5-10 years post enucleation. One of the advantages shown in this study when using different combined techniques, is that if you decompress or perform enucleation or marsupialization, the measures of the lesion first decrease, secondly, by having a reduction in the size of the lesion, the commitment of structures, however, reduces the risk of pathological fractures and even a wide resection, it should be noted that if they were injuries that do not respond to management, another should be performed, and consider when performing resection that a wide margin should be mentioned as mentioned by Pogrel $[6,8]$ Keratocysts can present satellite lesions, which would increase the risk of recurrence. Finally, it is highlighted that if the use of reconstruction materials is contemplated, such as in this case the titanium mesh (it served as support, containment, modeling, among others), the lyophilized bone placed allowed conduction, intervening and relating to the new formation, when performing enucleation removed the lesion and with the curettage the surgical area was stimulated inducing the formation of healthy bone, despite maintaining strict hygiene measures, the support of mouthwashes with chlorhexidine $0.12 \%$ (emphasizing it is an antiseptic widely used for oral cavity), the brushing technique that was taught to the patient and everything that involves a phase one, as well as the radiographic and clinical control, allowed the management to be successful.

\section{References}

1. Bava EJ, Ortolani A, Pantyrer M (2018) Multiple odontogenic keratocyst in a pediatric patient. Rev Asoc Odontol Argent 106(1): 35-40.

2. Meghanand TN, Anjali S, Abhishek S, Rohit S (2013) Odontogenic keratocyst: What is in the name? J Nat Sci Biol Med 4(2): 282-285.

3. Soluk Tekkesin M, Wright JM (2018) The world health organization classification of odontogenic lesions: A summary of the changes of the 2017, $4^{\text {th }}$ (Edn.), Turk J Pathology 34(1).

4. Pavelic B, Levanat S, Crnić I, Kobler P, Anić I, et al. (2001) PTCH gene altered in dentigerous cysts. J Oral Pathol Med 30(9): 569-576.

5. Wakolbinger R, Beck Mannagetta J (2016) Long-term results after treatment of extensive odontogenic cysts of the jaws: A review. Clin Oral Investig 20(1): 15-22.

6. Pogrel MA, Jordan RC (2004) Marsupialization as a definitive treatment for the odontogenic keratocyst. J Oral Maxillofac Surg 62(6): 651-655.

7. Martin L, Speight PM (2015) Odontogenic cysts. Diagnostic Histopathology 21(9): 359-369.

8. Pogrel M (2013) The Keratocystic Odontogenic Tumor. Oral Maxillofacial Surg Clin N Am 25(1): 21-30.

9. Motwani MB, Mishra SS, Anand RM, Degwekar SS, Bhowate RR (2011) Keratocystic odontogenic tumor: Case reports and review of literature. J Indian Acad Oral 
Open Access Journal of Dental Sciences

Med Radiol 23(2): 150-154.

10. Grasmuck EA, Nelson BL (2010) Keratocystic odontogenic tumor. Head Neck Pathol 4: 94-96.

11. Kramer IR, Pindborg JJ, Shear M (1992) Histological Typing of Odontogenic Tumors. Berlin, Heidelberg: Springer-Verlag, 37.
12. Giselle JRA, Francisco LS, Roberto OU, Daniel JR, Francisco GHJ, et al. (2020) Odontogenic Keratocyst and its Reduction by Decompressive Valve. Open Access J Dent Oral Surg 1(1): 1005.

13. Nair K, Lingappa A, Rangaiah P, Vittobarao P (2015) Keratocystic odontogenic tumor: A case report and review of literature. Journal of Indian Academy of Oral Medicine \& Radiology 27(2): 253-258. 